\title{
The NDT Development Process
}

\author{
M.J. Escalona, M. Mejías, J. Torres, and A. Reina \\ Department of Computer Languages and Systems \\ University of Seville \\ \{escalona, risoto, jtorres, reinaqu\}@lsi.us.es
}

\begin{abstract}
Web systems are more complex every day. This has produced that the research community poses the neccesity of developing methodological proposals which offer a suitable reference when a web information system is developed. Since the first proposal, HDM, was published [5] a lot of other methodologies have been developed in this environment. This paper presents NDT (Navigational Development Techniques) [3]. This proposal offers a different development process that starts with requirements treatment and allows to get design models using a systematic process.
\end{abstract}

\section{Introduction}

The progress of Internet and Communications has given way to a rising interest for developing methodological proposals. However, if these methodologies are analysed, most of them have a similar work process [7][1]. This paper presents a global vision of this classical process and presents some questions. After that, it offers another available vision to develop web information systems and it compares this new possibility with the classical process. In the third section, it presents a new methodological proposal called NDT (Navigational Development Techniques) that uses this new development process. Finally, in the last section some conclusions and future works are offered.

\section{The Development Process in Web Methodologies}

The number of methods, models and techniques that has appeared in web environment has produced that some comparative studies have been made in order to analyse similarities and differences between them [7][1]. Really, in web environment there is not a consensuos to model systems in the used terminology or in the used development process. Although there are different proposals, the most interesting aspect in our study is to analyse the development process that these methodologies use. The work of these methodologies is focussed on design which is mainly based on the conceptual model. This general characteristic is represented in figure 1 . As it is shown, the process starts with the requirements treatment. The group of analysts receive information from the users and customers that allows them to define the system requirements. Some methodologies do not cover this phase. Perhaps OOHDM [9], UWE [8] and UWA[11] are the most interested proposals in requirements treatment. Starting with them, the conceptual model of the system is designed. When this model is developed, other models like navigational model, abstract interface 
models, etc. are made. These other models are based on the conceptual model. Afterwards, the system implementation is realized using all these models. The user evaluated it and detects errors and bugs. This whole process is repeated until the suitable final system is obtained. However, although this process has been accepted, nowadays, there are some important points which are being questioned:

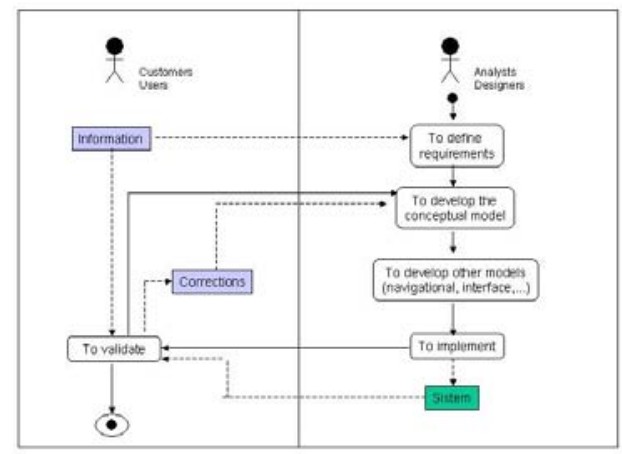

Fig. 1. The general development process in web methodologies

1. Uses cases as a technique to deal with web requirements: in the enumerated methodologies, the conceptual model is the base to define the other system models. Thus, the quality of the result depends on it. To get this model, web methodologies use the obtained information in the requirements treatment phase. This information is usually represented by using the use cases technique proposed in UML. However, several research groups [10][6] have commented that use cases can be a very ambiguous techinique to get the conceptual model easily. For this reason, new proposals are appearing like UIDs in OOHDM [10] or the proposal to refine requirements of UWA. These new proposals are oriented to define a more systematic procedure to get the conceptual model that guarantees its quality.

2. Separating concepts: since OOHDM proposed the division of concepts, the idea of modelling web aspects in an independent way has been hardly accepted [9][8][11]. Modelling these aspects separatelly, the developer can guarantee a cheaper maintenance and more possibilities for the system to be reused. However, this concept separation is being analysed nowadays [2]. There is also a strong dependence between conceptual models and the other ones which is produced directly from the development process (figure 1). To solve this dependence, some authors have classified requirements in different kinds, others propose to treat different requirements in a different way [4]. The question is that when the system is complex, it is not easy to get a conceptual model from the current requirements techniques systematically [4].

3. The communication with users and customers: from the comparative studies [7][1] it can be deduced that most web proposals do not include the user in the process. Web systems need that the development group has a huge knowledge about the system environment and users' neccesities [4]. The problem is how this communication must be done. The class diagram can be very difficult to be understood by some users. Use cases are very easy but they can sometimes be very abstract. The reality is that very few methodologies bear in mind this problem and 
only some of them offer proposals like reviews [4] or prototypes to make this communication easier.

4. Top-down process vs Bottom-up process: the process studied in the previous section can be defined as a top-down process. Starting with a general requirements definition, mainly made with use cases, a class conceptual model is designed. Opposite to this is the bottom-up process. It is based on doing a very exhaustive study in the first phases and after that, on getting the other models. In table 1, their advantages(+) and disadvantages(-) are enumerated. Obviously, to select one process or another is a development team's decision. Although the most applied tendency is a top-down process [9][8], some new proposals are oriented to a bottom-up process [3][11].

Table 1. Advantages and disadvantages in bottom-up and top-down process

\begin{tabular}{l|l} 
Top-down process & Bottom-up process \\
\hline + Requirements definition is often cheaper and easier. & -Requirements definition is often more complex \\
\hline + The communication with users is often easier. & $\begin{array}{l}\text { - Sometimes, it is difficult to confront all requirements } \\
\text { specification since the beginning. }\end{array}$ \\
$\begin{array}{l}\text { - To get the conceptual model is more complex and it } \\
\text { is necessary to interact with users constantly. }\end{array}$ & $\begin{array}{l}\text { + The conceptual model is made in a more systematic } \\
\text { way. }\end{array}$ \\
\hline $\begin{array}{l}\text { - Other models depend on the conceptual model. The } \\
\text { separation of concepts is based on this model. }\end{array}$ & $\begin{array}{l}\text { + The separation of concepts is made in requirements, so } \\
\text { models are more independent. }\end{array}$ \\
\hline - Mistakes and errors are detected in late phases & + Mistakes and errors are detected in the first phases \\
\hline
\end{tabular}

\section{NDT Development Process}

According to the previous section, NDT development process can be defined as a bottom-up process. The development process is focused on a very detail requirements definition guided by objectives, which covers three subphases: requirements capture, requirements definition and requirements validation. In figure 2 , it is presented.

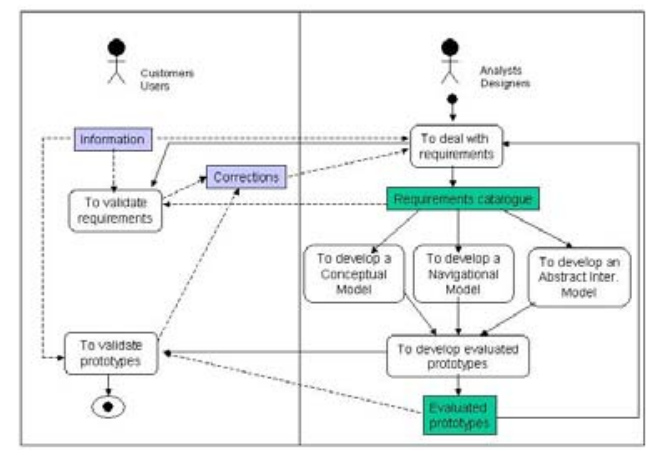

Fig. 2. NDT development process

It is a bottom-up process where models are independent. The process starts by defining objectives. Using a described procedure, requirements are captured and defined. Requirements are classified and dealt with depending on whether they are: information storage requirements, interaction requirements, etc. When requirements 
are validated, the NDT process continues defining three models: the conceptual model, the navigational model and the abstract interface model. The most important characteristic of the NDT process is that the way from requirements to models is systematic and makes each model independent. It is systematic because NDT offers algorithms which indicate how each model has to be obtained from the requirements definition. And they are independent because, although different models are related between them, because all of them represent an aspect of the same system, each of them can be obtained independently from the requirements. From these models, some evaluated prototypes can be obtained. These prototypes can be evaluated by users and customers. In this section, the NDT process is finished. The main objective of NDT is to offer a systematic process to get models which other accepted web methodologies use as the base to their development process and to guarantee the quality of these models. NDT is not a new web methodological proposal. It is a procedure to get design models from the users' requirements. As it has been indicated, NDT starts with a requirements definition and gets design models from them in a systematic way. The fact that this process can be carried out systematically allows to translate this systematic process in an automatic process using a case tool. Thus, a tool which lets execute the NDT process, apply its techniques and get its results is being implemented. Nowadays, only the requirements treatment phase is implemented, but we are working in order to include in NDT-Tool the whole process of NDT.

\section{Conclusions and Future Works}

This paper has presented a very general vision of NDT, a new methodological proposition to get requirements and design models in a systematic way in web information systems. A general description of web methodologies and their general development process has been offered. Also, some important aspects in this general process like the communication with users or the dependency in the conceptual model has been analyzed. After that, bottom-up and top-down development processes have been compared and some advantages and disadvantages have been enumerated. Base on this comparison, a global vision of NDT has been shown. Finally, a short presentation of NDT-Tool, the NDT case tool, has been done. One of the most important advantages of NDT is that it has being applied to several real examples, like the system to manage information about historical inheritance in Andalusia or the system to manage public subventions in the public cultural administration in Andalusia. These and other projects have been developed in collaboration with public and private companies getting very good results. As a future work, we want to finish developing the NDT process and, obviously, to implement completely NDT-Tool in order to cover the whole NDT life cycle.

\section{References}

1 Barry, C. \& Lang, M. A Survey of Multimedia and Web Development Tecniques and Methodology Usage. IEEE Multimedia. April-June 2001, 52-56

2 Cachero, C. , Koch, N. Conceptual Navigation Analysis : a Device and Plataform Independent Navigation Specification. $2^{\text {nd }}$ IWOST. June, 2002.

3 Escalona, M.J.,Mejías, M., Torres, J. Reina.A. NDT: Una técnica para el desarrollo de la navegación. Congreso IDEAS 2002. Cuba. Abril 2002 
4 Escalona, M.J., Koch, N. Ingeniería de Requisitos en Aplicaciones para la Web- Un estudio comparativo. Department of Language and Computer Science. December, 2002.

5 Garzoto F., Schwabe D. and Paolini P. HDM-A Model Based Approach to Hypermedia Aplication Design. ACM Trnasactions on Information System, 11 (1), pp 1-26. 1993.

6 Insfrán, E., Pastor, O., Wieringa, R. Requirements Engineering-Based Conceptual Modeling. Requirements Engineering Journal, Vol 7 (1). 2002.

7 Koch, N. A comparative study of methods for Hypermedia Development. Technical Report 9905. Ludwig-Maximilian-University, Munich, Germany. 1999.

8 Koch, N. Software Engineering for Adaptative Hypermedia Applications. Ph. Thesis, FAST Reihe Softwaretechnik Vol(12), Uni-Druck Publishing Company, Germany. 2001.

9 G. Rossi An Object Oriented Method for Designing Hipermedia Applications. PHD Thesis, Departamento de Informática, PUC-Rio, Brazil, 1996

10 Vilain, P., Schwabe, D., Sieckenius, C. (2000). A diagrammatic Tool for Representing User Interaction in UML. Lecture Notes in Computer Science. Proc. UML'2000. York.

11 UWA Consortium, UWA Requirements Elicitation: Model, Notation, and Tool Architecture. 